\title{
Recent developments for regular functions of a hypercomplex variable
}

\author{
Graziano Gentili, Caterina Stoppato, Daniele C. Struppa and Fabio \\ Vlacci
}

\begin{abstract}
In this paper we survey a series of recent developments in the theory of functions of a hypercomplex variable. The central idea underlying these developments consists in requiring a function to be holomorphic on suitable slices of the space on which the function itself is defined. Specifically, we apply this approach to functions defined on the space $\mathbb{H}$ of quaternions, on the space $\mathbb{O}$ of octonions, and finally on the Clifford algebra of type $(0,3)$, denoted $C l(0,3)$. The properties of these functions resemble those of holomorphic functions, and yet the different nature of the three algebras on which we work introduces new and exciting phenomena.
\end{abstract}

Mathematics Subject Classification (2000). Primary: 30G35, 32W05.

Keywords. functions of hypercomplex variables, $\bar{\partial}$-type operators.

\section{Introduction}

In the last couple of years, the authors developed a new theory of regularity for functions defined on the algebras of quaternions and octonions, as well as on an eight dimensional Clifford algebra, $[17,18,19,20]$. The new definition of regularity is quite simple: it only requires holomorphicity on complex slices of the algebra under consideration. Despite its simplicity, the theory ends up being quite powerful in replicating the fundamental results of the theory of holomorphic functions of a complex variable. More importantly, polynomial functions as well as convergent power series can be treated in this setting, unlike what happens in the usual theories of monogenic and Fueter-regular functions. This aspect of the theory is

This work was partially supported by GNSAGA of the INdAM and by PRIN "Proprietà geometriche delle varietà reali e complesse" and "Geometria Differenziale e Analisi Globale" of the MIUR. 


\section{Graziano Gentili, Caterina Stoppato, Daniele C. Struppa and Fabio Vlacci}

quite relevant because it can be applied to the study of a functional calculus in a non-commutative setting, $[3,4,5,6]$. In this survey paper, we highlight in a unified fashion the fundamental ideas of the theory, and we anticipate a few results which have not been published yet. We want to thank the anonymous referee, whose comments have significantly improved the final version of this paper.

\section{Hamilton, Cayley and Clifford Algebras}

It is very rare, in mathematics, to have a clear and explicit description of the birth and the origin of a new theory: in most cases, in fact, it is even difficult to identify a date which could be taken as its birthday. Luckily, the situation is different for the birth of the theory of quaternions as well as for the theory of octonions ${ }^{1}$.

Sir William R. Hamilton was looking for ways of extending complex numbers (which can be viewed as points on the 2-dimensional Gauss plane) to higher spatial dimensions. He could not do so for dimension 3, and in fact it was later shown by Frobenius that this task is actually impossible: the only associative division algebras which are finite dimensional over the real numbers are the real numbers

1

Extract from a letter from Sir W. R. Hamilton to Professor P. G. Tait.

Letter dated October 15, 1858.

. . . P.S. - To-morrow will be the 15th birthday of the Quaternions. They started into life, or light, full grown, on [Monday] the 16th of October, 1843, as I was walking with Lady Hamilton to Dublin, and came up to Brougham Bridge, which my boys have since called the Quaternion Bridge. That is to say, I then and there felt the galvanic circuit of thought close; and the sparks which fell from it were the fundamental equations between $i, j, k$; exactly such as I have used them ever since. I pulled out on the spot a pocket-book, which still exists, and made an entry, on which, at the very moment, I felt that it might be worth my while to expend the labour of at least ten (or it might be fifteen) years to come. But then it is fair to say that this was because I felt a problem to have been at that moment solved - an intellectual want relieved which had haunted me for at least fifteen years before.

Less than an hour elapsed before I had asked and obtained leave of the Council of the Royal Irish Academy, of which Society I was, at that time, the President - to read at the next General Meeting a Paper on Quaternions; which I accordingly did, on November 13, 1843.

Some of those early communications of mine to the Academy may still have some interest for a person like you, who has since so well studied my volume, which was not published for ten years afterwards.

In the meantime, will you not do honour to the birthday to-morrow, in an extra cup of - ink? for it may be obsolete now to propose XXX, or even XYZ.

Note: Robert P. Graves notes in his biography of Hamilton that 'Brougham Bridge', referred to by Hamilton in his letters regarding the discovery of quaternions, is properly referred to as Broome Bridge: so called from the name of a family residing near.

Source: [23] 
$\mathbb{R}$, the complex numbers $\mathbb{C}$ and the non-commutative algebra $\mathbb{H}$ of quaternions or Hamilton numbers. Furthermore, the only non-associative division algebra which is finite dimensional over the real numbers is the algebra $\mathbb{O}$ of octonions or Cayley numbers (see, e.g., [1, 22]). Eventually Hamilton tried dimension 4 and created quaternions $^{2}$.

${ }^{2}$ According to Hamilton, on October 16 he was out walking along the Royal Canal in Dublin with his wife when the solution in the form of the equation

$$
i^{2}=j^{2}=k^{2}=i j k=-1
$$

suddenly occurred to him; Hamilton then promptly carved this equation into the side of the nearby Broom Bridge (which Hamilton called Brougham Bridge). Since 1989, the National University of Ireland, Maynooth has organized a pilgrimage, where mathematicians take a walk from Dunsink observatory to the bridge where, unfortunately, no trace of the carving remains, though a stone plaque does commemorate the discovery.

Letter from Sir W. R. Hamilton to Rev. Archibald H. Hamilton.

Letter dated August 5, 1865.

My dear Archibald - (1) I had been wishing for an occasion of corresponding a little with you on QUATERNIONS: and such now presents itself, by your mentioning in your note of yesterday, received this morning, that you "have been reflecting on several points connected with them" (the quaternions), "particularly on the Multiplication of Vectors." (2) No more important, or indeed fundamental question, in the whole Theory of Quaternions, can be proposed than that which thus inquires What is such MULTIPLICATION? What are its Rules, its Objects, its Results? What Analogies exist between it and other Operations, which have received the same general Name? And finally, what is (if any) its Utility? (3) If I may be allowed to speak of myself in connexion with the subject, I might do so in a way which would bring you in, by referring to an ante-quaternionic time, when you were a mere child, but had caught from me the conception of a Vector, as represented by a Triplet: and indeed I happen to be able to put the finger of memory upon the year and month - October, 1843 - when having recently returned from visits to Cork and Parsonstown, connected with a meeting of the British Association, the desire to discover the laws of the multiplication referred to regained with me a certain strength and earnestness, which had for years been dormant, but was then on the point of being gratified, and was occasionally talked of with you. Every morning in the early part of the above-cited month, on my coming down to breakfast, your (then) little brother William Edwin, and yourself, used to ask me, "Well, Papa, can you multiply triplets"? Whereto I was always obliged to reply, with a sad shake of the head: "No, I can only add and subtract them." (4) But on the 16th day of the same month which happened to be a Monday, and a Council day of the Royal Irish Academy - I was walking in to attend and preside, and your mother was walking with me, along the Royal Canal, to which she had perhaps driven; and although she talked with me now and then, yet an under-current of thought was going on in my mind, which gave at last a result, whereof it is not too much to say that I felt at once the importance. An electric circuit seemed to close; and a spark flashed forth, the herald (as I foresaw, immediately) of many long years to come of definitely directed thought and work, by myself if spared, and at all events on the part of others, if I should even be allowed to live long enough distinctly to communicate the discovery. Nor could I resist the impulse - unphilosophical as it may have been - to cut with a knife on a stone of Brougham Bridge, as we passed it, the fundamental formula with the symbols, $i, j, k$; namely, $i^{2}=j^{2}=k^{2}=i j k=-1$ which contains the Solution of the Problem, but of course, as an inscription, has long since mouldered away. A 


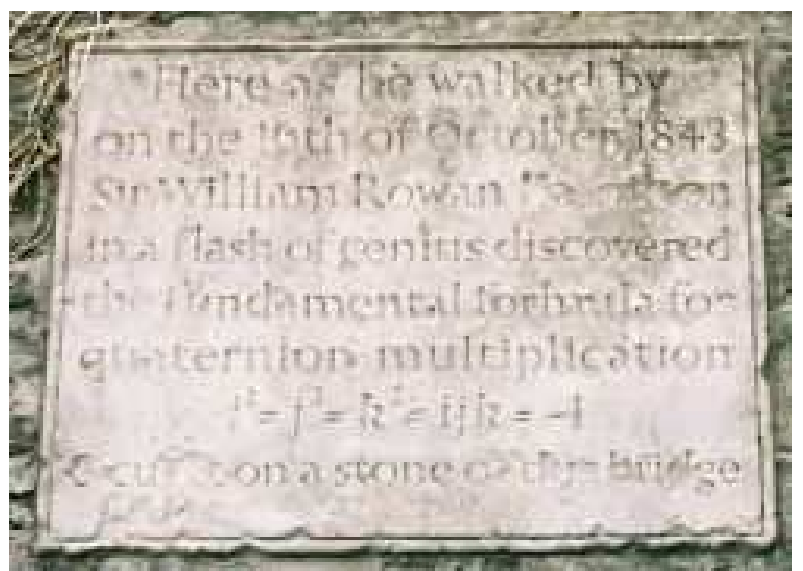

Commemorating stone plaque on Broom Bridge

The quaternions are of the form $q=x_{0}+i x_{1}+j x_{2}+k x_{3}$ where the $x_{l}$ are real $(l=0, \ldots, 3)$, and $i, j, k$, are imaginary units (i.e. their square equals -1 ) such that, according to $(1), i j=-j i=k, j k=-k j=i$, and $k i=-i k=j$. In this way, $\mathbb{H}$ can be considered as a vector space over the real numbers of dimension 4 . Notice that a generic element $q$ of $\mathbb{H}$ can be written as

$$
q=\left(x_{0}+x_{1} i\right)+\left(x_{2}+x_{3} i\right) j
$$

a fact that allows an identification of $\mathbb{H}$ with pairs of complex numbers, each in $\mathbb{R}+\mathbb{R} i$; this split $\mathbb{H}=\mathbb{C}+\mathbb{C} j$ will play a crucial role in the next section.

We quoted explicitly from Hamilton's letters to P. G. Tait (dated October 15, 1858) and to his son A.H.Hamilton (dated August 5, 1865), but an even more important correspondence took place on October 17, 1843, when Hamilton described his discovery of quaternions to his good friend John T. Graves [24]. As Baez points out in [1], it was Graves' interest in algebra that stimulated some of Hamilton's own work in this direction. Soon after Hamilton discovered the quaternions, Graves developed his own theory of octonions (which he called octaves and described in a letter to Hamilton, dated December 26, 1843). Interestingly enough, octonions are now associated to the name of Cayley, because Graves did not publish his discovery, while Cayley did in March, 1845.

more durable notice remains, however, on the Council Books of the Academy for that day (October 16th, 1843), which records the fact, that I then asked for and obtained leave to read a Paper on Quaternions, at the First General Meeting of the session: which reading took place accordingly, on Monday the 13th of the November following. With this quaternion of paragraphs I close this letter I.; but I hope to follow it up very shortly with another.

Your affectionate father, William Rowan HAMILTON.

Source: [23] 
The algebra $\mathbb{O}$ of octonions can be described in a similar way to what we have done for quaternions. Consider a basis $\mathcal{E}=\left\{e_{0}=1, e_{1}, \ldots, e_{6}, e_{7}\right\}$ of $\mathbb{R}^{8}$ and relations

$$
e_{\alpha} e_{\beta}=-\delta_{\alpha \beta}+\psi_{\alpha \beta \gamma} e_{\gamma}, \quad \alpha, \beta, \gamma=1,2, \ldots, 7
$$

where $\delta_{\alpha \beta}$ is the Kronecker delta and $\psi_{\alpha \beta \gamma}$ equals 1 when $(\alpha, \beta, \gamma)$ is one of the following combinations

$$
(1,2,3),(1,4,5),(2,4,6),(3,4,7),(2,5,7),(1,6,7),(5,3,6),
$$

it is totally antisymmetric in $\alpha, \beta, \gamma$, and it equals 0 in the remaining cases. The generic element of $\mathbb{O}$ can be written as

$$
w=x_{0}+x_{1} e_{1}+\ldots+x_{7} e_{7} .
$$

but one can show that $\left(1, e_{1}, e_{2}, e_{1} e_{2}\right)$ form a basis for a subalgebra of $\mathbb{O}$ isomorphic to the algebra $\mathbb{H}$ of quaternions. In fact we have the decomposition

$$
\begin{gathered}
\mathbb{O}=\left(\mathbb{R}+\mathbb{R} e_{1}\right)+\left(\mathbb{R}+\mathbb{R} e_{1}\right) e_{2}+\left[\left(\mathbb{R}+\mathbb{R} e_{1}\right)+\left(\mathbb{R}+\mathbb{R} e_{1}\right) e_{2}\right] e_{4}= \\
=\mathbb{C}+\mathbb{C} e_{2}+\left(\mathbb{C}+\mathbb{C} e_{2}\right) e_{4}=\mathbb{H}+\mathbb{H} e_{4} .
\end{gathered}
$$

Given a generic element $w$ of $\mathbb{H}$ or $\mathbb{O}$ we define in a natural fashion its conjugate $\bar{w}=x_{0}-\sum_{k \geq 1} x_{k} e_{k}$, and its square norm $|w|^{2}=w \bar{w}=\sum_{k \geq 0} x_{k}^{2}$. The following is immediate and yet important

Proposition 2.1. For any non-real quaternion or octonion $w$, there exist, and are unique, $x, y \in \mathbb{R}$ with $y>0$, and an imaginary unit $I_{w}$ such that $w=x+y I_{w}$.

Definition 2.2. Given any imaginary unit $I$, the set $\mathbb{R}+\mathbb{R} I$ will be denoted by $L_{I}$.

Notice that after identifying the imaginary unit $I_{w}$ in $\mathbb{H}$ or in $\mathbb{O}$ with the imaginary unit $i$ of $\mathbb{C}$, the set $L_{I_{w}}$ may be considered as a complex plane in $\mathbb{H}$ or (1) passing through 0,1 and $w$. In this way both $\mathbb{H}$ and $\mathbb{O}$ can be obtained as an infinite union of complex planes (which will be also called slices). Now, let $C l(0,3)$ denote the real Clifford algebra of signature $(0,3)$. This algebra can be defined as follows (see [8] for this and other related definitions): let $E=\left\{e_{1}, e_{2}, e_{3}\right\}$ be the canonical orthonormal basis for $\mathbb{R}^{3}$ with defining relations $e_{i} e_{j}+e_{j} e_{i}=-2 \delta_{i j}$. An element of the Clifford algebra $C l(0,3)$ can be written in a unique way as

$$
w=x_{0}+x_{1} e_{1}+x_{2} e_{2}+x_{3} e_{3}+x_{12} e_{1} e_{2}+x_{13} e_{1} e_{3}+x_{23} e_{2} e_{3}+x_{123} e_{1} e_{2} e_{3}
$$

where the coefficients $x_{i}, x_{i j}, x_{i j k}$ are real numbers. Thus, we see that $C l(0,3)$ is an eight dimensional real space, endowed with a natural multiplicative structure. Notice that the square of each unit $e_{i}, e_{i} e_{j}$ is -1 , while the square of $e_{1} e_{2} e_{3}$ is 1 . For this reason, the element $e_{1} e_{2} e_{3}$ is often referred to as a pseudoscalar. Therefore it is appropriate to define the set of Clifford real numbers as $\mathcal{R}=\left\{w=x_{0}+x_{123} e_{1} e_{2} e_{3}\right\}$ and the set of Clifford imaginary numbers as $\mathcal{I}=\left\{w=x_{1} e_{1}+x_{2} e_{2}+x_{3} e_{3}+\right.$ $\left.x_{12} e_{1} e_{2}+x_{13} e_{1} e_{3}+x_{23} e_{2} e_{3}\right\}$. With these definitions, we can decompose any element $w$ of $C l(0,3)$ as the sum of an element in $\mathcal{R}$, its real part $\operatorname{Re}(w)$, and an element in $\mathcal{I}$, its imaginary part $\operatorname{Im}(w)$. 
6 Graziano Gentili, Caterina Stoppato, Daniele C. Struppa and Fabio Vlacci

Let $\mathbb{A}$ denote any of the algebras $\mathbb{C}, \mathbb{H}, \mathbb{O}, C l(0,3)$. For each of these algebras, we can define the set of roots of -1 as $\mathbb{S}_{\mathbb{A}}=\left\{w \in \mathbb{A}: w^{2}=-1\right\}$. This set will be referred to as the sphere of imaginary units of $\mathbb{A}$. A second set of interest is what we call the unit imaginary sphere, namely $\mathbb{U}_{\mathbb{A}}=\{w \in \mathbb{A}: \operatorname{Re}(w)=0,|\operatorname{Im}(w)|=1\}$. The identity between $\mathbb{S}_{\mathbb{A}}$ and $\mathbb{U}_{\mathbb{A}}$ which holds for $\mathbb{A}=\mathbb{C}, \mathbb{H}, \mathbb{O}$ can be easily verified (see $[17,18,19]$ ) and implies that $\mathbb{S}_{\mathbb{C}}, \mathbb{S}_{\mathbb{H}}$ and $\mathbb{S}_{\mathbb{O}}$ are, respectively, 0-dimensional, 2-dimensional and 6-dimensional spheres.

On the other hand (see [20]), an element $w=x_{0}+x_{1} e_{1}+x_{2} e_{2}+x_{3} e_{3}+$ $x_{12} e_{1} e_{2}+x_{13} e_{1} e_{3}+x_{23} e_{2} e_{3}+x_{123} e_{1} e_{2} e_{3}$ in $C l(0,3)$ belongs to $\mathbb{S}_{C l(0,3)}$ if and only if it belongs to $\mathbb{U}_{C l(0,3)}$ and its coordinates satisfy

$$
x_{1} x_{23}-x_{2} x_{13}+x_{3} x_{12}=0 .
$$

Moreover, it turns out that the set of $w \in C l(0,3)$ such that $w^{2}=1$ reduces to $w= \pm 1$ and $w= \pm e_{1} e_{2} e_{3}$.

If one were to attempt to replicate proposition 2.1 in the Clifford algebra $C l(0,3)$, one would need to be able to write every element $w$ of $C l(0,3)$ as the sum of a Clifford real number and the product of an element of $\mathbb{S}_{C l(0,3)}$ by a Clifford real number, namely

$$
w=\left(\alpha+\beta e_{1} e_{2} e_{3}\right)+I\left(\gamma+\delta e_{1} e_{2} e_{3}\right)
$$

where $\alpha, \beta, \gamma, \delta$ are real numbers and $I \in \mathbb{S}_{C l(0,3)}$. This is not always possible. Indeed, denote by $\mathcal{U}$ the set of all Clifford numbers $w=x_{0}+x_{1} e_{1}+x_{2} e_{2}+$ $x_{3} e_{3}+x_{12} e_{1} e_{2}+x_{13} e_{1} e_{3}+x_{23} e_{2} e_{3}+x_{123} e_{1} e_{2} e_{3}$ in $C l(0,3)$ such that $w \in \mathcal{R}$ or $\left(x_{1}, x_{2}, x_{3}\right) \neq \pm\left(x_{23},-x_{13}, x_{12}\right)$. In [20] we proved

Proposition 2.3. Let $w \in C l(0,3)$. There exist $I \in \mathbb{S}_{C l(0,3)}$ and $\alpha, \beta, \gamma, \delta \in \mathbb{R}$ such that $w=\left(\alpha+\beta e_{1} e_{2} e_{3}\right)+I\left(\gamma+\delta e_{1} e_{2} e_{3}\right)$ if and only if $w \in \mathcal{U}$. The above representation is unique up to substituting $(I, \gamma, \delta)$ by $-(I, \gamma, \delta)$ or $\pm\left(I_{1} e_{2} e_{3}, \delta, \gamma\right)$.

\section{Regular Functions}

Since the beginning of last century, mathematicians have been interested in creating a theory of quaternion valued functions of a quaternionic variable, which would somehow resemble the classical theory of holomorphic functions of one complex variable. The simplest extension, namely the request for a quaternionic function to have a quaternionic derivative, fails to be of any interest since (see [37])

Proposition 3.1. Let $\Omega$ be a simply-connected domain in $\mathbb{H}$ and let $f: \Omega \rightarrow \mathbb{H}$. If for any $q_{0} \in \Omega$

$$
\lim _{q \rightarrow q_{0}}\left(q-q_{0}\right)^{-1}\left(f(q)-f\left(q_{0}\right)\right)
$$

exists in $\mathbb{H}$, then necessarily $f(q)=q a+b$ for some $a, b \in \mathbb{H}$. If for any $q_{0} \in \Omega$

$$
\lim _{q \rightarrow q_{0}}\left(f(q)-f\left(q_{0}\right)\right)\left(q-q_{0}\right)^{-1}
$$

exists in $\mathbb{H}$, then necessarily $f(q)=a q+b$ for some $a, b \in \mathbb{H}$. 
Another quite natural approach could be to consider the class of functions which admit (local) series expansions of the form

$$
\sum_{s} m_{s}\left(q-q_{0}\right)
$$

with $m_{s}(q)$ finite sum of monomials of the type $a_{0} \cdot q \cdot a_{1} \ldots a_{s-1} \cdot q \cdot a_{s}$. But if $q=x_{0}+i x_{1}+j x_{2}+k x_{3}$, then it is very easy to verify that $x_{0}=\frac{1}{4}(q-i q i-j q j-k q k)$, $x_{1}=\frac{1}{4 i}(q-i q i+j q j+k q k), x_{2}=\frac{1}{4 j}(q+i q i-j q j+k q k), x_{3}=\frac{1}{4 k}(q+i q i+j q j-k q k)$, so that the class of maps considered coincides with the class of real analytic maps of $\mathbb{R}^{4}$ in $\mathbb{R}^{4}$.

In the 1930s, Fueter (see $[12,13]$; for a modern and more recent approach see also [33]) introduced the differential operator

$$
\frac{\partial}{\partial \bar{q}}=\frac{1}{4}\left(\frac{\partial}{\partial x_{0}}+i \frac{\partial}{\partial x_{1}}+j \frac{\partial}{\partial x_{2}}+k \frac{\partial}{\partial x_{3}}\right)
$$

now known as the Cauchy-Fueter operator and defined the space of regular functions as the space of solutions of the equation associated to this operator. This theory of regular functions is very well developed, in many different directions, and we refer the reader to [37] for the basic features of these functions. For more recent work in this area we refer the reader to $[8,27,29,30]$ and references therein. Furthermore, an analogue to the Cauchy-Fueter operator, called the Dirac operator, has been defined in Clifford algebras (see [8]); in this environment, any solution of the Dirac operator is known as a monogenic function.

While the theory of Fueter-regular functions is extremely successful in replicating many important properties of holomorphic functions (and not only in one variable, see [8]), even the simplest quaternionic polynomials fail to be regular in the sense of Fueter. Another unexpected consequence in the definition of regular functions in the sense of Fueter is that their zero-sets can vary a lot and have real dimension zero, one, two or four (see [37]).

Following an idea of Cullen [11] two of us were inspired to give the following definition (see $[17,18,19])$ :

Definition 3.2. Let $\mathbb{K}$ be either $\mathbb{H}$ or $\mathbb{O}$. If $\Omega$ is a domain in $\mathbb{K}$, a real differentiable function $f: \Omega \rightarrow \mathbb{K}$ is said to be Cullen-regular if, for every $I \in \mathbb{S}_{\mathbb{K}}$, its restriction $f_{I}$ to the complex line $L_{I}=\mathbb{R}+\mathbb{R} I$ passing through the origin and containing 1 and $I$ is holomorphic on $\Omega \cap L_{I}$.

Throughout the paper, since no confusion can arise, we will refer to Cullenregular functions as regular functions tout court. Note that in our most recent work we have used the expression slice regular instead.

In the spirit of Gateaux, a notion of $I$-derivative is defined as follows:

Definition 3.3. Let $\Omega$ be a domain in $\mathbb{K}$ and let $f: \Omega \rightarrow \mathbb{K}$ be a real differentiable function. For any $I \in \mathbb{S}_{\mathbb{K}}$ and any point $q=x+y I$ in $\Omega$ ( $x$ and $y$ are real numbers 
8 Graziano Gentili, Caterina Stoppato, Daniele C. Struppa and Fabio Vlacci here) we define the $I$-derivative of $f$ at $q$ as

$$
\partial_{I} f(x+y I):=\frac{1}{2}\left(\frac{\partial}{\partial x}-I \frac{\partial}{\partial y}\right) f_{I}(x+y I)
$$

The definition of regularity extends to the case of $C l(0,3)$ as follows (see [20]). Recall that $\mathcal{U}=\left\{w=x_{0}+x_{1} e_{1}+x_{2} e_{2}+x_{3} e_{3}+x_{12} e_{1} e_{2}+x_{13} e_{1} e_{3}+x_{23} e_{2} e_{3}+\right.$ $x_{123} e_{1} e_{2} e_{3}$ in $C l(0,3)$ such that $w \in \mathcal{R}$ or $\left.\left(x_{1}, x_{2}, x_{3}\right) \neq \pm\left(x_{23},-x_{13}, x_{12}\right)\right\}$.

Definition 3.4. Let $\Omega$ be a domain in $\mathcal{U}$. A real differentiable function $f: \Omega \rightarrow$ $C l(0,3)$ is said to be regular if, for every $I \in \mathbb{S}_{C l(0,3)}$, its restriction $f_{I}$ to the four dimensional plane $L_{I}=\mathcal{R}+I \mathcal{R}=\left\{\left(t_{1}+t_{2} e_{1} e_{2} e_{3}\right)+I\left(t_{3}+t_{4} e_{1} e_{2} e_{3}\right)\right\}$ passing through the origin and containing 1 and $I$ satisfies, on $\Omega \cap L_{I}$, the system

$$
2 D_{I} f_{I}:=\left(d_{12}+I d_{34}\right) f_{I}=0
$$

where $d_{i j}=d_{t_{i} t_{j}}$ indicates the (real) differential with respect to the variables $t_{i}$ and $t_{j}$.

Fix $I \in \mathbb{S}_{C l(0,3)}$ and let $K=I e_{1} e_{2} e_{3}$. Each $w \in L_{I}=L_{K}$ can be represented in the following two ways

$$
\begin{aligned}
& w=\left(t_{1}+t_{2} e_{1} e_{2} e_{3}\right)+I\left(t_{3}+t_{4} e_{1} e_{2} e_{3}\right)=\left(t_{1}+I t_{3}\right)+\left(t_{2}+I t_{4}\right) e_{1} e_{2} e_{3} \\
& w=\left(t_{1}+t_{2} e_{1} e_{2} e_{3}\right)+K\left(t_{4}+t_{3} e_{1} e_{2} e_{3}\right)=\left(t_{1}+K t_{4}\right)+\left(t_{2}+K t_{3}\right) e_{1} e_{2} e_{3} .
\end{aligned}
$$

Chosen $J \in \mathbb{S}_{C l(0,3)}$, we can represent $f_{I}=f_{K}$ (see [20]) either as

$$
\begin{aligned}
f_{I} & =\left(f_{00}+f_{01} e_{1} e_{2} e_{3}\right)+I\left(f_{10}+f_{11} e_{1} e_{2} e_{3}\right)+\left[\left(g_{00}+g_{01} e_{1} e_{2} e_{3}\right)+I\left(g_{10}+g_{11} e_{1} e_{2} e_{3}\right)\right] J= \\
& =\left(f_{00}+I f_{10}\right)+\left(f_{01}+I f_{11}\right) e_{1} e_{2} e_{3}+\left[\left(g_{00}+I g_{10}\right)+\left(g_{01}+I g_{11}\right) e_{1} e_{2} e_{3}\right] J= \\
& =F_{0}+F_{1} e_{1} e_{2} e_{3}+\left(G_{0}+G_{1} e_{1} e_{2} e_{3}\right) J
\end{aligned}
$$

or as

$$
\begin{aligned}
f_{K} & =\left(f_{00}+f_{01} e_{1} e_{2} e_{3}\right)+K\left(f_{11}+f_{10} e_{1} e_{2} e_{3}\right)+\left[\left(g_{00}+g_{01} e_{1} e_{2} e_{3}\right)+K\left(g_{11}+g_{10} e_{1} e_{2} e_{3}\right)\right] J= \\
& =\left(f_{00}+K f_{11}\right)+\left(f_{01}+K f_{10}\right) e_{1} e_{2} e_{3}+\left[\left(g_{00}+K g_{11}\right)+\left(g_{01}+K g_{10}\right) e_{1} e_{2} e_{3}\right] J= \\
& =M_{0}+M_{1} e_{1} e_{2} e_{3}+\left(N_{0}+N_{1} e_{1} e_{2} e_{3}\right) J .
\end{aligned}
$$

The previous equations imply that $2 D_{I} f_{I}=\left(d_{12}+I d_{34}\right) f_{I}=0$ if, and only if,

$$
\begin{gathered}
\left\{\begin{array} { l } 
{ d _ { 1 2 } f _ { 0 0 } = d _ { 3 4 } f _ { 1 0 } } \\
{ d _ { 1 2 } f _ { 1 0 } = - d _ { 3 4 } f _ { 0 0 } }
\end{array} \quad \left\{\begin{array}{l}
d_{12} f_{01}=d_{34} f_{11} \\
d_{12} f_{11}=-d_{34} f_{01}
\end{array}\right.\right. \\
\left\{\begin{array} { l } 
{ d _ { 1 2 } g _ { 0 0 } = d _ { 3 4 } g _ { 1 0 } } \\
{ d _ { 1 2 } g _ { 1 0 } = - d _ { 3 4 } g _ { 0 0 } }
\end{array} \quad \left\{\begin{array}{l}
d_{12} g_{01}=d_{34} g_{11} \\
d_{12} g_{11}=-d_{34} g_{01}
\end{array}\right.\right.
\end{gathered}
$$


and $2 D_{K} f_{K}=\left(d_{12}+K d_{43}\right) f_{K}=0$ if, and only if,

$$
\begin{gathered}
\left\{\begin{array} { l } 
{ d _ { 1 2 } f _ { 0 0 } = d _ { 4 3 } f _ { 1 1 } } \\
{ d _ { 1 2 } f _ { 1 1 } = - d _ { 4 3 } f _ { 0 0 } }
\end{array} \quad \left\{\begin{array}{l}
d_{12} f_{01}=d_{43} f_{10} \\
d_{12} f_{10}=-d_{43} f_{01}
\end{array}\right.\right. \\
\left\{\begin{array} { l } 
{ d _ { 1 2 } g _ { 0 0 } = d _ { 4 3 } g _ { 1 1 } } \\
{ d _ { 1 2 } g _ { 1 1 } = - d _ { 4 3 } g _ { 0 0 } }
\end{array} \quad \left\{\begin{array}{l}
d_{12} g_{01}=d_{43} g_{10} \\
d_{12} g_{10}=-d_{43} g_{01} .
\end{array}\right.\right.
\end{gathered}
$$

The two-dimensional Cauchy-Riemann systems (8) and (9) are compatible (i.e. they share solutions) and it turns out that the set of regular functions is not empty (see [20]).

We conclude this section recalling the following representations of $f_{I}$, which derive from the geometric properties of imaginary units shown in the first section. We will often refer to these results as the Splitting Lemmas (see [17, 18, 19]).

Lemma 3.5. If $f$ is a regular function on $\Omega \subset \mathbb{K}$, then for every $I_{1} \in \mathbb{S}_{\mathbb{K}}$

1. if $\mathbb{K}=\mathbb{H}$, for any $J \in \mathbb{S}_{\mathbb{H}}, J \perp I_{1}$, there exist two holomorphic functions $F, G: \Omega \cap L_{I_{1}} \rightarrow L_{I_{1}}$ such that

$$
f_{I_{1}}(z)=F(z)+G(z) J
$$

2. if $\mathbb{K}=\mathbb{O}$ and $I_{2}, I_{4} \in \mathbb{S}_{\mathbb{O}}$ are properly chosen, (see [18]), then there exist four holomorphic functions $F_{1}, F_{2}, G_{1}, G_{2}: \Omega \cap L_{I_{1}} \rightarrow L_{I_{1}}$ such that

$$
f_{I_{1}}(z)=F_{1}(z)+F_{2}(z) I_{2}+\left(G_{1}(z)+G_{2}(z) I_{2}\right) I_{4} .
$$

In the case of $C l(0,3)$ we have (see [20])

Lemma 3.6. Let $\Omega$ be a domain in $\mathcal{U} \subseteq C l(0,3)$ and let $f$ be regular in $\Omega$. Fix $I \in \mathbb{S}_{C l(0,3)}$, set $K=I e_{1} e_{2} e_{3}$. According to equations (5), each $w \in L_{I}=L_{K}$ can be represented as $w=z_{1}+z_{2} e_{1} e_{2} e_{3}$ with $z_{1}, z_{2} \in \mathbb{R}+I \mathbb{R} \simeq \mathbb{C}$ and as $w=$ $\zeta_{1}+\zeta_{2} e_{1} e_{2} e_{3}$ with $\zeta_{1}, \zeta_{2} \in \mathbb{R}+K \mathbb{R} \simeq \mathbb{C}$.

Chosen $J \in \mathbb{S}_{C l(0,3)}$ there exist $F_{0}, F_{1}, G_{0}, G_{1}$ holomorphic in $\left(z_{1}, z_{2}\right)$ and $M_{0}, M_{1}, N_{0}, N_{1}$ holomorphic in $\left(\zeta_{1}, \zeta_{2}\right)$ (see [20]) such that

$f_{I}=F_{0}+F_{1} e_{1} e_{2} e_{3}+\left(G_{0}+G_{1} e_{1} e_{2} e_{3}\right) J=M_{0}+M_{1} e_{1} e_{2} e_{3}+\left(N_{0}+N_{1} e_{1} e_{2} e_{3}\right) J=f_{K}$ as in equations (6) and (7).

\section{Power Series and Series Expansions for Regular Functions}

Given $a \in \mathbb{K}$, the basic monomial $w^{n} a$ defines a regular function in $\mathbb{K}$ according to Definition 3.2. Since the sum of regular functions is regular, we immediately have that polynomials with coefficients in $\mathbb{K}$ on the right side are regular. Moreover, since convergence of power series is uniform on compact sets, it turns out that power series $\sum_{n=0}^{+\infty} w^{n} a_{n}$ with coefficients in $\mathbb{K}$ are also regular in their domain of convergence, which proves to be a ball $B(0, R)=\{w \in \mathbb{K}:|w|<R\}$. The following result is proven in [19]: 
Theorem 4.1. A function $f: B=B(0, R) \rightarrow \mathbb{K}$ is regular if, and only if, it has a series expansion of the form

$$
f(q)=\sum_{n=0}^{+\infty} q^{n} \frac{1}{n !} \frac{\partial^{n} f}{\partial x^{n}}(0)
$$

converging in B. In particular if $f$ is regular then it is $C^{\infty}$ in $B$.

Recall that (see [20]) for an arbitrary point $w$ in $\mathcal{U}$, there exist $I \in \mathbb{S}_{C l(0,3)}$ and $z_{1}, z_{2} \in \mathbb{R}+I \mathbb{R}$ such that $w=z_{1}+z_{2} e_{1} e_{2} e_{3}$. With this in mind, we can state the analogue of Theorem 4.1 in $C l(0,3)$.

Theorem 4.2. Let $f$ be a regular function in $\mathcal{U}$. For each choice of $I \in \mathbb{S}_{C l(0,3)}$ and for all $z_{1}, z_{2} \in \mathbb{R}+I \mathbb{R}$ we have

$$
f\left(z_{1}+z_{2} e_{1} e_{2} e_{3}\right)=\sum_{m, n \in \mathbb{N}} z_{1}^{m} z_{2}^{n} \frac{\partial^{m+n} f(0)}{\partial z_{1}^{m} \partial z_{2}^{n}} .
$$

The power series expansion which we have obtained for regular functions is the key ingredient in proving the analogues of many well known results of holomorphic functions in one complex variable. For this reason, in what follows we will always restrict our attention to functions which are regular in a ball $B(0, R)$ centered at the origin of $\mathbb{K}(\mathbb{K}=\mathbb{H}$ or $\mathbb{K}=\mathbb{O})$.

Remark 4.3. The forthcoming book [7] will study regular quaternionic functions on the larger class of slice domains, namely those domains $\Omega \subseteq \mathbb{H}$ such that $\Omega \cap \mathbb{R}$ is non-empty and, for any $I \in \mathbb{S}, L_{I} \cap \Omega$ is a domain in $L_{I}$.

\section{Cauchy Formula and Related Results}

We will now list some interesting results for regular functions. The proofs, which are contained in [18, 19], mainly involve the Splitting Lemmas and techniques which are imported from the theory of holomorphic functions of one complex variable. The notations are the same as in the previous sections. We begin with this version of the identity principle.

Theorem 5.1. Let $f$ and $g$ be regular functions on $B=B(0, R)$. If there exists $I \in \mathbb{S}_{\mathbb{K}}$ such that $f \equiv g$ on a subset of $L_{I} \cap B$ having an accumulation point in $L_{I} \bigcap B$, then $f \equiv g$ in $B$.

The following versions of the mean value property and the maximum modulus principle hold for regular functions.

Proposition 5.2. If $f: B=B(0, R) \rightarrow \mathbb{K}$ is a regular function, $I \in \mathbb{S}_{\mathbb{K}}$ and $a \in B \cap L_{I}$, then, for any $r>0$ small enough,

$$
f(a)=\frac{1}{2 \pi} \int_{0}^{2 \pi} f_{I}\left(a+r e^{I \vartheta}\right) d \vartheta
$$


Theorem 5.3. Let $f: B=B(0, R) \rightarrow \mathbb{K}$ be a regular function. If $|f|$ has a maximum at $a \in B$, then $f$ is constant in $B$.

Perhaps the most important consequence of the Splitting Lemma 3.5 is the analogue, for regular functions, of the Cauchy representation formula. In order to state it appropriately, we will adopt the following notation. If $w \in \mathbb{K}$, we set

$$
I_{w}=\left\{\begin{array}{l}
\frac{\operatorname{Im}(w)}{|\operatorname{Im}(w)|} \in \mathbb{S}_{\mathbb{K}} \quad \text { if } \operatorname{Im}(w) \neq 0 \\
\text { any element of } \mathbb{S}_{\mathbb{K}} \text { otherwise. }
\end{array}\right.
$$

Notice that for any $\zeta \in L_{I_{w}}, \zeta \neq w$ the equality $(\zeta-w)^{-1} d \zeta=d \zeta(\zeta-w)^{-1}$ holds. We can now state this integral representation formula for regular functions.

Theorem 5.4. Let $f: B=B(0, R) \rightarrow \mathbb{K}$ be a regular function, and let $w \in B$. Then

$$
f(w)=\frac{1}{2 \pi I_{w}} \int_{\partial \Delta_{I_{w}}(0, r)} \frac{d \zeta}{(\zeta-w)} f(\zeta)
$$

where $\zeta \in L_{I_{w}} \cap B$, and where $r>0$ is such that

$$
\overline{\Delta_{I_{w}}(0, r)}=\left\{x+y I_{w}: x^{2}+y^{2} \leq r^{2}\right\}
$$

is contained in $B$ and $w \in \Delta_{I_{w}}(0, r)$.

As a consequence we obtain:

Theorem 5.5 (Cauchy Estimates). Let $f: B=B(0, R) \rightarrow \mathbb{K}$ be a regular function, let $r<R, I \in \mathbb{S}_{\mathbb{K}}$, and $\partial \Delta_{I}(0, r)=\left\{(x+y I): x^{2}+y^{2}=r^{2}\right\}$. If $M_{I}=\max \{|f(w)|$ : $\left.w \in \partial \Delta_{I}(0, r)\right\}$ and if $M=\inf \left\{M_{I}: I \in \mathbb{S}_{\mathbb{K}}\right\}$ then, for all $n \geq 0$,

$$
\frac{1}{n !}\left|\frac{\partial^{n} f}{\partial x^{n}}(0)\right| \leq \frac{M}{r^{n}}
$$

We now state the analogue of the Liouville theorem.

Theorem 5.6. Let $f: \mathbb{K} \rightarrow \mathbb{K}$ be an entire regular map (i.e. a regular map defined and regular everywhere on $\mathbb{K})$. If $f$ is bounded, i.e. there exists a positive number $M$ such that $|f(w)| \leq M$ for all $w \in \mathbb{K}$, then $f$ is constant.

We close this section with this version of Morera's theorem.

Theorem 5.7. Let $f: B=B(0, R) \rightarrow \mathbb{K}$ be a differentiable function. If, for every $I \in \mathbb{S}_{\mathbb{K}}$, the differential form $f(z) d z, z=x+y I, x, y \in \mathbb{R}$, defined on $L_{I} \cap B$ is closed, then the function $f$ is regular. 
12 Graziano Gentili, Caterina Stoppato, Daniele C. Struppa and Fabio Vlacci

\section{The Schwarz Lemma of the Unit Ball and its Boundary Generalizations}

Before focussing our attention on other peculiar properties of regular functions, we want to devote this section to some rigidity properties shared by regular and holomorphic functions (see - for the holomorphic case -, e.g., [2, 26, 31, 38]; for the regular case we refer to [21]).

We begin by stating an analogue of the classical Schwarz lemma for regular functions: its proof (see $[18,19]$ ) is not a consequence of the Splitting Lemmas. The argument relies upon the fact that a regular function has a power series expansion.

Theorem 6.1. Let $B(0,1)=\{w \in \mathbb{K}:|w|<1\}$. If $f: B(0,1) \rightarrow B(0,1)$ is a regular function such that $f(0)=0$. Then for every $w \in B$,

$$
|f(w)| \leq|w|
$$

and

$$
\left|f^{\prime}(0)\right| \leq 1
$$

The statement of Theorem 6.1 with equality in (11) can be considered as a Cartan-type result (see [39]) for regular functions in the ball $B(0,1)$. Generalizing it to the case of a generic fixed point $w_{0} \in B(0,1)$ is not easy. First of all, Moebius transformations (in the sense of [25]) are not regular in general: in [18, 19] we proved that

Proposition 6.2. For any $w_{0} \in B(0,1) \backslash \mathbb{R}$, the map $\eta$ defined by

$$
\eta(w)=\left(w-w_{0}\right)\left(1-\overline{w_{0}} w\right)^{-1}
$$

is a diffeomorphism of $B(0,1)$ onto itself but it is not regular.

This problem is addressed, in the case of quaternions, in the forthcoming paper [16], where the authors study the new class of regular Moebius transformations. It is also possible to transform the unit ball biregularly into the the right half space $\mathbb{K}^{+}=\{w \in \mathbb{K}: \operatorname{Re}(w)>0\}$ : if we define the Cayley transformation as $\psi(w)=(1-w)^{-1}(1+w)$, then $($ see $[18,19])$

Lemma 6.3. The Cayley transformation $\psi: B(0,1) \rightarrow \mathbb{K}^{+}$is a one-to-one regular function of $w$ with (regular) inverse the function $\phi(w)=(w-1)(w+1)^{-1}$.

Nevertheless, since the regularity of functions is not preserved under composition, the above mentioned Cayley transformation and regular Moebius transformations do not help in generalizing theorem 6.1. The following is indeed proven by using a different technique (see [21]):

Theorem 6.4. Assume that $f: B(0,1) \rightarrow B(0,1)$ is a regular function and there exists $w_{0} \in B(0,1)$ such that $f\left(w_{0}\right)=w_{0}$ and that $f^{\prime}\left(w_{0}\right)=1$. Then $f(w)=w$ for every $w \in B(0,1)$.

An interesting result, which can be regarded as transposition of a result due to Rudin (see [35]) is the following 
Theorem 6.5. Let $f$ be a regular self-map of the unit ball $B(0,1)$ of $\mathbb{K}$ and suppose there exists $r \in \mathbb{R}$ such that $f(r)=r$. Then either $f$ has no other fixed points in $B(0,1)$ or $f$ is the Identity of $B(0,1)$.

We now want to consider "boundary" generalizations of Schwarz-Cartan type Theorems in the setting of regular functions. For this purpose, we will start by recalling (see [2])

Theorem 6.6. Let $\Delta$ be the open unit disc of $\mathbb{C}$ and let $f: \Delta \rightarrow \Delta$ be holomorphic and such that

as $z \rightarrow 1$. Then $f \equiv I d_{\Delta}$.

$$
f(z)=1+(z-1)+o\left(|z-1|^{3}\right)
$$

This (sharp) result, which has been extensively used to obtain rigidity results for holomorphic self-maps in some pseudoconvex domains of $\mathbb{C}^{n}$ (see $[2,26]$ ), is achieved by applying Herglotz representation formula and Hopf's Lemma. In [38] the authors use a more direct approach to prove a generalization of Theorem 6.6 which gives some insight for other rigidity results. For the regular case in [21] the following analogue of Theorem 6.6 is obtained

Theorem 6.7. Let $f$ be a regular self-map of $B(0,1) \subset \mathbb{K}$. Assume there exists $w_{0} \in \partial B(0,1)$ such that

$$
f(w)=w_{0}+\left(w-w_{0}\right)+o\left(\left|w-w_{0}\right|^{3}\right)
$$

as $w \rightarrow w_{0}$. Then $f \equiv I d_{B(0,1)}$.

\section{Zeroes of Regular Functions}

In this section, we recall the main results which give a complete description of the zero-sets for regular quaternionic functions; quite surprisingly, these zero-sets have a structure which turns out to be significantly different from that of Fueter-regular functions or holomorphic functions of two complex variables.

Furthermore, the approach used for regular power series offers a shorter proof of the results stated for polynomials in [32]. From now on we will denote $\mathbb{S}_{\mathbb{H}}$ by $\mathbb{S}$. The crucial and key point in the description of the zero-set of a regular function is the following (see $[17,18,19]$ )

Theorem 7.1. Let $\sum_{n=0}^{+\infty} q^{n} a_{n}$ be a given quaternionic power series with radius of convergence $R$. Suppose that there exist $x_{0}, y_{0} \in \mathbb{R}$ and $I, J \in \mathbb{S}$ with $I \neq J$ such that

and

$$
\sum_{n=0}^{+\infty}\left(x_{0}+y_{0} I\right)^{n} a_{n}=0
$$

$$
\sum_{n=0}^{+\infty}\left(x_{0}+y_{0} J\right)^{n} a_{n}=0 .
$$


14 Graziano Gentili, Caterina Stoppato, Daniele C. Struppa and Fabio Vlacci

Then for all $L \in \mathbb{S}$ we have

$$
\sum_{n=0}^{+\infty}\left(x_{0}+y_{0} L\right)^{n} a_{n}=0 .
$$

The main result which describes the geometric properties of the zero-set is the following (see [15])

Theorem 7.2 (Structure of the Zero-Set). Let $f$ be a regular function on an open ball $B(0, R)$ centered in the origin of $\mathbb{H}$. If $f$ is not identically zero then its zero-set $Z_{f}$ consists of isolated points or isolated spheres of the form $x+y \mathbb{S}$, for $x, y \in \mathbb{R}$, $y \neq 0$.

This result has a curious consequence concerning the zeroes of holomorphic functions. Since (the power series expansion of) any holomorphic function $f$ can be uniquely extended to (the power series expansion of) a regular function $\widetilde{f}$ over quaternions, the question of distinguishing which zeroes of $f$ will remain isolated after the extension, and which will become "spherical", naturally arises. It turns out that each pair of conjugate zeroes of $f$ contributes a spherical zero of $\widetilde{f}$, while the other zeroes of $f$ correspond to isolated zeroes of $\widetilde{f}$. The techniques employed to prove theorem 7.2 suggest the use of the following multiplication between regular power series.

Definition 7.3. Let $f(q)=\sum_{n=0}^{+\infty} q^{n} a_{n}$ and $g(q)=\sum_{n=0}^{+\infty} q^{n} b_{n}$ be given quaternionic power series with radii of convergence greater than $R$. We define the regular product of $f$ and $g$ as the series $f * g(q)=\sum_{n=0}^{+\infty} q^{n} c_{n}$, where $c_{n}=\sum_{k=0}^{n} a_{k} b_{n-k}$ for all $n$.

We point out that the sequence of the coefficients of the regular product $f * g$ is the discrete convolution of the sequences of the coefficients of $f$ and $g$. In the polynomial case, the regular multiplication concides with the classical multiplication of the polynomial ring over the quaternions, $\mathbb{H}[X]$. In the sequel, $h^{* n}=h * \ldots * h$ will denote the $n$-th power of a regular function $h$ with respect to $*$-multiplication. We have the following result.

Theorem 7.4. Let $f: B(0, R) \rightarrow \mathbb{H}$ be a regular function and let $p$ belong to $B(0, R)$. Then $f(p)=0$ if and only if there exists a regular function $g: B(0, R) \rightarrow \mathbb{H}$ such that $f(q)=(q-p) * g(q)$.

Furthermore we can describe the zero-set of a regular product in terms of the zero-sets of the factors:

Theorem 7.5 (Zeros of a Regular Product). Let $f, g: B(0, R) \rightarrow \mathbb{H}$ be regular and $p \in B(0, R)$. Then $f * g(p)=0$ if and only if $f(p)=0$ or $f(p) \neq 0$ and $g\left(f(p)^{-1} p f(p)\right)=0$.

This theorem extends to quaternionic power series the theory presented in [28] for polynomials. 


\section{Poles of Regular Functions}

Given the peculiar properties of the zeros which are summarized in the previous section, a new question arises. Do regular functions have singularities resembling the poles of holomorphic complex functions? This question receives a positive answer in [36]:

Proposition 8.1. Consider a quaternionic Laurent series $f(q)=\sum_{n \in \mathbb{Z}} q^{n} a_{n}$ with quaternionic coefficients $a_{n} \in \mathbb{H}$. There exists a spherical shell $A=A\left(0, R_{1}, R_{2}\right)=$ $\left\{q \in \mathbb{H}: R_{1}<|q|<R_{2}\right\}$ such that: (i) the series $f^{+}(q)=\sum_{n=0}^{+\infty} q^{n} a_{n}$ and $f^{-}(q)=$ $\sum_{n=1}^{+\infty} q^{-n} a_{-n}$ both converge absolutely and uniformly on the compact subsets of $A$; (ii) $f^{+}(q)$ diverges for $|q|>R_{2}$; (iii) $f^{-}(q)$ diverges for $|q|<R_{1}$. If $A$ is not empty (i.e. $0 \leq R_{1}<R_{2}$ ) then the function $f: A \rightarrow \mathbb{H}$ defined by $f(q)=\sum_{n \in \mathbb{Z}} q^{n} a_{n}=$ $f^{+}(q)+f^{-}(q)$ is regular.

This allows the construction of functions which are regular on a punctured ball $B(0, R) \backslash\{0\}$ and have a singularity at 0 . Moreover, any function which is regular on a spherical shell $A\left(0, R_{1}, R_{2}\right)$ admits a Laurent series expansion centered at 0 . The latter is a special case of the following.

Theorem 8.2. Let $f$ be a regular function on a domain $\Omega$, let $p \in \mathbb{H}$ and let $L_{I}$ be a complex line through $p$. If $\Omega$ contains an annulus $A_{I}=A\left(p, R_{1}, R_{2}\right) \cap L_{I}$ then there exists $\left\{a_{n}\right\}_{n \in \mathbb{Z}} \subseteq \mathbb{H}$ such that $f_{I}(z)=\sum_{n \in \mathbb{Z}}(z-p)^{n} a_{n}$ for all $z \in A_{I}$. If, moreover, $p \in \mathbb{R}$ then $f(q)=\sum_{n \in \mathbb{Z}}(q-p)^{n} a_{n}$ for all $q \in A\left(p, R_{1}, R_{2}\right) \cap \Omega$.

Definition 8.3. Let $f, p$ and $\left\{a_{n}\right\}_{n \in \mathbb{Z}}$ be as in theorem 8.2. The point $p$ is called a pole if there exists an $n \in \mathbb{N}$ such that $a_{-m}=0$ for all $m>n$; the minimum of such $n \in \mathbb{N}$ is called the order of the pole and denoted as ord $f_{f}(p)$. If $p$ is not a pole for $f$ then we call it an essential singularity for $f$.

Notice that, by the final statement of theorem 8.2, real singularities are completely analogous to singularities of holomorphic functions of one complex variable and there is no resemblance to the case of several complex variables. As for nonreal singularities, theorem 8.2 only provides information on the complex line $L_{I}$ through the point $p$; we apparently cannot predict the behavior of the function in a (four-dimensional) neighborhood of $p$. In order to overcome this difficulty, we need to introduce a couple of new definitions.

Definition 8.4. For a regular function $f: B(0, R) \rightarrow \mathbb{H}$ having power series expansion $\sum_{n=0}^{+\infty} q^{n} a_{n}$ we define the regular conjugate $f^{c}$ and the symmetrization 
16 Graziano Gentili, Caterina Stoppato, Daniele C. Struppa and Fabio Vlacci

$f^{s}$ of $f$ as $f^{c}(q)=\sum_{n=0}^{+\infty} q^{n} \bar{a}_{n}, f^{s}(q)=f * f^{c}(q)=f^{c} * f(q)=\sum_{n=0}^{+\infty} q^{n} r_{n}$ with $r_{n}=\sum_{k=0}^{n} a_{k} \bar{a}_{n-k} \in \mathbb{R}$.

Definition 8.5. Let $f, g: B=B(0, R) \rightarrow \mathbb{H}$ be regular functions and suppose $f \not \equiv 0$. The (left) regular quotient of $f$ and $g$ is the function $f^{-*} * g$ defined on $B \backslash Z_{f^{s}}$ by $f^{-*} * g(q)=\frac{1}{f^{s}(q)} f^{c} * g(q)$. Moreover, the regular reciprocal of $f$ is the function $f^{-*}=f^{-*} * 1$.

Regular quotients are regular on their domains of definition, and the algebraic meaning of this construction is explained by the following result.

Proposition 8.6. Fix $R$ and consider the associative real algebra $\left(\mathcal{D}_{R},+, *\right)$ of regular functions on $B(0, R)$. If we endow the set of left regular quotients $\mathcal{L}_{R}=$ $\left\{f^{-*} * g: f, g \in \mathcal{D}_{R}, f \not \equiv 0\right\}$ with the multiplication $*$ defined by $\left(f^{-*} * g\right) *\left(h^{-*} * k\right)=$ $\frac{1}{f^{s} h^{s}} f^{c} * g * h^{c} * k$ then $\left(\mathcal{L}_{R},+, *\right)$ is a division algebra over $\mathbb{R}$ and it is the classical ring of quotients of $\left(\mathcal{D}_{R},+, *\right)$.

For the definition of the classical ring of quotients, see [34]. Regular quotients allow a detailed study of the poles. Define a function $f$ semiregular if it does not have essential singularities or, equivalently, if the restriction $f_{I}$ is meromorphic for all $I \in \mathbb{S}$. As proven in [36], $f$ is semiregular on $B\left(0, R_{0}\right)$ if and only if $f_{\left.\right|_{B(0, R)}}$ is a left regular quotient for all $R<R_{0}$. This allows the definition of a multiplication operation $*$ on the set of semiregular functions on a ball and the proof of the following result.

Theorem 8.7. Let $f$ be a semiregular function on $B=B(0, R)$, choose $p \in B$ and let $m=\operatorname{ord}_{f}(p), n=\operatorname{ord}_{f}(\bar{p})$. There exists a unique semiregular function $g$ on $B$ such that

$$
f(q)=\left[(q-p)^{* m} *(q-\bar{p})^{* n}\right]^{-*} * g(q)
$$

The function $g$ is regular near $p$ and $\bar{p}$ and $g(p) \neq 0, g(\bar{p}) \neq 0$, provided $m>0$ or $n>0$.

The previous result allows the study of the structure of the poles.

Theorem 8.8 (Structure of the Poles). If $f$ is a semiregular function on $B=$ $B(0, R)$ then $f$ extends to a regular function on $B$ minus a union of isolated real points or isolated 2-spheres of the type $x+y \mathbb{S}=\{x+y I: I \in \mathbb{S}\}$ with $x, y \in \mathbb{R}, y \neq 0$. All the poles on each 2-sphere $x+y \mathbb{S}$ have the same order with the possible exception of one, which must have lesser order.

\section{The Open Mapping Theorem}

One of the most celebrated properties of holomorphic functions of one complex variable is the fact that they are open. In this section we will present a version of the open mapping theorem for regular quaternionic functions, which states that 
each such function is open when restricted to an appropriate open subset of its domain of definition.

The regular quotients introduced in the previous section allow the proof of the minimum modulus principle for regular quaternionic functions, which leads to the open mapping theorem. The first step in this direction is taken by proving the following relation between the regular quotient $f^{-*} * g(q)$ and the quotient $f(q)^{-1} g(q)=\frac{1}{f(q)} g(g)($ see $[14,36])$.

Theorem 9.1. Let $f, g$ be regular functions on $B=B(0, R)$. Setting $T_{f}(q)=$ $f^{c}(q)^{-1} q f^{c}(q)$ defines a diffeomorphism of $B \backslash Z_{f^{s}}$ onto itself, with inverse function $T_{f}^{-1}=T_{f^{c}}$. Moreover,

for all $q \in B \backslash Z_{f s}$.

$$
f^{-*} * g(q)=\frac{1}{f\left(T_{f}(q)\right)} g\left(T_{f}(q)\right)
$$

Let $f: B=B(0, R) \rightarrow \mathbb{H}$ be a regular function. Applying the maximum modulus principle to the regular reciprocal $f^{-*}(q)=\frac{1}{f\left(T_{f}(q)\right)}$ proves that, if $|f|$ has a local minimum point $p=x+y I \in B$, then either $f$ is constant or $f$ has a zero in $x+y \mathbb{S}$. This is not enough for our proof of the open mapping theorem, which requires the following (stronger) property.

Theorem 9.2 (Minimum Modulus Principle). Let $f: B=B(0, R) \rightarrow \mathbb{H}$ be $a$ regular function. If $|f|$ has a local minimum point $p \in B$ then either $f(p)=0$ or $f$ is constant.

The proof is given in [14] and depends on the following peculiar property of regular functions.

Theorem 9.3. Let $f: B=B(0, R) \rightarrow \mathbb{H}$ be a regular function. For all $x, y \in \mathbb{R}$ such that $x+y \mathbb{S} \subseteq B$ there exist $b, c \in \mathbb{H}$ such that $f(x+y I)=b+$ Ic for all $I \in \mathbb{S}$.

In other words, when restricted to a 2 -sphere $x+y \mathbb{S}$, a regular function is either constant or an affine map of $x+y \mathbb{S}$ onto a 2 -sphere $b+\mathbb{S} c$ with $b, c \in \mathbb{H}$.

Example 9.4. Consider the polynomial function $f(q)=q^{2}$. For $x, y \in \mathbb{R}$ we have $f(x+y I)=x^{2}-y^{2}+I 2 x y$ for all $I \in \mathbb{S}$. Thus $f$ maps each 2 -sphere $x+y \mathbb{S}$ with $y \neq 0$ onto $\left(x^{2}-y^{2}\right)+\mathbb{S}(2 x y)$. In particular, if $x=0$ then $f$ is constant on $x+y \mathbb{S}=y \mathbb{S}$.

Proposition 9.5. If $f: B=B(0, R) \rightarrow \mathbb{H}$ is a regular function, denote by $D_{f}$ the union of all the 2-spheres $x+y \mathbb{S}$ (for $x, y \in \mathbb{R}, y \neq 0$ ) on which $f$ is constant. Then $D_{f}$, which we call the degenerate set of $f$, is closed in $B \backslash \mathbb{R}$. Moreover, if $f$ is not constant then the interior of $D_{f}$ is empty.

We can now state the main result of [14].

Theorem 9.6 (Open Mapping Theorem). Let $f: B(0, R) \rightarrow \mathbb{H}$ be a non-constant regular function and let $D_{f}$ be its degenerate set. Then $f: B(0, R) \backslash \overline{D_{f}} \rightarrow \mathbb{H}$ is open. 
18 Graziano Gentili, Caterina Stoppato, Daniele C. Struppa and Fabio Vlacci

As already mentioned, the proof is based on the minimum modulus principle. Theorem 9.6 is a sharp result: the function $f(q)=q^{2}$ in example 9.4 proves to be open on $\mathbb{H} \backslash \overline{D_{f}}=\mathbb{H} \backslash\{q \in \mathbb{H}: \operatorname{Re}(q)=0\}$ but not on $\mathbb{H}$. Nevertheless, theorem 9.6 extends as follows. We say that $U \subseteq \mathbb{H}$ is circular if, for all $x+y I \in U$ with $y \neq 0$, the whole 2 -sphere $x+y \mathbb{S}$ is contained in $U$.

Theorem 9.7. Let $f: B(0, R) \rightarrow \mathbb{H}$ be a regular function. If $U$ is a circular open subset of $B(0, R)$, then $f(U)$ is open. In particular $f(B(0, R))$ is an open set.

\section{Conclusions}

Every time mathematicians define a new concept, two questions arise naturally. The first is whether the new concept can lead to an interesting theory by itself. We believe that the previous sections have demonstrated that the notion of sliceregularity is indeed a very stable notion, that suitably generalizes the fundamental properties of holomorphicity to the hypercomplex setting. At the same time, we identified some crucial differences between the complex and the hypercomplex case. The second question is whether the new theory, in addition to its intrinsic value, can also contribute to the solution of some outstanding problem. Two of us, with different coauthors, prove that this is the case in [6]. This paper offers a survey of how the ideas discussed in this paper have been applied to develop two new functional calculi in non-commutative settings. Those calculi are based on the notion of slice regularity and a similar notion of slice monogenicity. In addition to [6], the reader interested in the details is referred to $[3,4,5,7,9]$, and [10].

\section{References}

[1] J. Baez, The octonions. Bull. Amer. Math. Soc. 39 (2002), 145-205.

[2] D. M. Burns, S. G. Krantz, Rigidity of holomorphic mappings and a new Schwarz lemma at the boundary. J. Amer. Math. Soc. 7 (1994), 661-676.

[3] F. Colombo, G. Gentili, I. Sabadini, D. C. Struppa, A functional calculus in a non commutative setting. Electron. Res. Announc. Math. Sci., 14 (2007), 60-68.

[4] F. Colombo, G. Gentili, I. Sabadini, D. C. Struppa, Non commutative functional calculus: bounded operators, E-print. arXiv:0708.3591v1 [math.SP]

[5] F. Colombo, G. Gentili, I. Sabadini, D. C. Struppa, Non commutative functional calculus: unbounded operators, E-print. arXiv:0708.3592v2 [math.SP]

[6] F. Colombo, G. Gentili, I. Sabadini, D. C. Struppa, An overview of functional calculus in different settings, this volume.

[7] F. Colombo, G. Gentili, I. Sabadini, D. C. Struppa, Functional Calculus in a Noncommutative Setting, in preparation.

[8] F. Colombo. I. Sabadini, F. Sommen, D. C. Struppa, Analysis of Dirac systems and computational algebra. Birkhäuser, Boston, 2004.

[9] F. Colombo, I. Sabadini, D. C. Struppa, A new functional calculus for non commuting operators. J. Funct. Anal., 254 (2008), 2255-2274. 
[10] F. Colombo, I. Sabadini, D. C. Struppa, Slice monogenic functions. To appear in Isr. J. Math.

[11] C. G. Cullen, An integral theorem for analytic intrinsic functions on quaternions. Duke Math. J. 32 (1965), 139-148.

[12] R. Fueter, Die Funktionentheorie der Differentialgleichungen $\triangle u=0$ und $\triangle \triangle u=0$ mit vier reellen Variablen. Comm. Math. Helv. 7 (1934), 307-330.

[13] R. Fueter, Über einen Hartogs'schen Satz. Comment. Math. Helv. 12 (1939/40), 7580.

[14] G. Gentili, C. Stoppato, The open mapping theorem for quaternionic regular functions, E-print. arXiv:0802.3861v1 [math.CV]

[15] G. Gentili, C. Stoppato, Zeros of regular functions and polynomials of a quaternionic variable. To appear in Michigan Math. J.

[16] G. Gentili, C. Stoppato, Regular Moebius transformations of the space of quaternions, in preparation.

[17] G. Gentili, D. C. Struppa, A new approach to Cullen-regular functions of a quaternionic variable. C. R. Acad. Sci. Paris, Ser. I 342 (2006), 741-744.

[18] G. Gentili, D. C. Struppa, Regular functions on the space of Cayley numbers, Preprint. Dipartimento di Matematica "U. Dini", Università di Firenze, n. 13, (2006).

[19] G. Gentili, D. C. Struppa, A new theory of regular functions of a quaternionic variable. Adv. Math. 216 (2007), 279-301.

[20] G. Gentili, D. C. Struppa, Regular functions on a Clifford Algebra. Complex Var. Elliptic Equ. 53 (2008), no. 5, 475-483.

[21] G. Gentili, F. Vlacci, Rigidity for regular functions over Hamilton and Cayley numbers and a boundary Schwarz' Lemma. To appear in Indag. Mathem.

[22] R. Godement, Cours d'algèbre. Herman, Paris, 1978.

[23] R. P. Graves, Life of Sir William Rowan Hamilton, Arno Press, 1975. Volume II, Chapter XXVIII

[24] W. R. Hamilton, Copy of a letter from Sir William R. Hamilton to John T. Graves, Esq. Philosophical Magazine, 3rd series, 25 1844, 489-495.

[25] R. Heidrich, G. Jank, On the Iteration of Quaternionic Moebius Transformations. Complex Var. Theory Appl. 29 (1996), no. 4, 313-318.

[26] X. Huang, A boundary rigidity problem for holomorphic mappings on some weakly pseudoconvex domains. Can. J. Math. 47 (2) (1995), 405-420.

[27] V. V. Kravchenko, M. Shapiro, Integral Representations for Spatial Models of Mathematical Physics. Pitman Res. Notes in Math., 351. Longman, Harlow, 1996.

[28] T. Y. Lam, A first course in noncommutative rings. Graduate Texts in Mathematics, 123. Springer-Verlag, New York, 1991. 261-263.

[29] M.E. Luna-Elizarrarás, M.A. Macías-Cedeño, M. V. Shapiro, On some relations between the derivative and the two-dimensional directional derivatives of a quaternionic function. AIP Conference Procedings, 936, 2007, 758-760.

[30] M.E. Luna-Elizarrarás, M.A. Macías-Cedeño, M. V. Shapiro, On the derivatives of quaternionic functions along two-dimensional planes. To appear in Adv. Appl. Clifford Algebr. 
20 Graziano Gentili, Caterina Stoppato, Daniele C. Struppa and Fabio Vlacci

[31] S. Migliorini, F. Vlacci, A new rigidity result for holomorphic maps. Indag. Mathem. (N. S.) 13 (2002), no. 4, 537-549.

[32] A. Pogorui, M. V. Shapiro, On the structure of the set of zeros of quaternionic polynomials. Complex Var. Theory Appl. 49 (2004), no. 6, 379-389.

[33] T. Qian, F. Sommen, Deriving harmonic functions in higher dimensional spaces, $Z$. Anal. Anwendungen 22 (2003), no. 2, 275-288.

[34] L. H. Rowen, Ring theory. Student edition. Academic press, San Diego, 1991. 272279.

[35] W. Rudin, Function theory in the unit ball of $\mathbb{C}^{n}$. Springer, Berlin, 1980.

[36] C. Stoppato, Poles of regular quaternionic functions, E-print. arXiv:0804.1665v1 [math.CV]

[37] A. Sudbery, Quaternionic analysis. Math. Proc. Camb. Phil. Soc. 85 (1979), 199-225.

[38] R. Tauraso, F. Vlacci, Rigidity at the boundary for self-maps of the unit disc. Complex Var. Theory Appl. 45 (2001), no. 2, 151-165.

[39] E. Vesentini, Capitoli scelti della teoria delle funzioni olomorfe. Unione Matematica Italiana, Bologna, 1984.

Graziano Gentili

Dipartimento di Matematica "U. Dini" - Università di Firenze

Viale Morgagni, 67/A, 50134 Firenze, Italy

e-mail: gentili@math.unifi.it

Caterina Stoppato

Dipartimento di Matematica "U. Dini" - Università di Firenze

Viale Morgagni, 67/A, 50134 Firenze, Italy

e-mail: stoppato@math.unifi.it

Daniele C. Struppa

Department of Mathematics and Computer Science

Schmid College of Science - Chapman University

One University Drive, Orange, CA 92866 USA

e-mail: struppa@chapman.edu

Fabio Vlacci

Dipartimento di Matematica "U. Dini" - Università di Firenze

Viale Morgagni, 67/A, 50134 Firenze, Italy

e-mail: vlacci@math.unifi.it 